ANNALES

POLONICI MATHEMATICI

LXXVII.2 (2001)

\title{
Multiplicity of positive solutions for a nonlinear fourth order equation
}

\author{
by D. R. Dunninger (East Lansing, MI)
}

\footnotetext{
Abstract. We study the existence and multiplicity of positive solutions of the nonlinear fourth order problem

$$
\left\{\begin{array}{l}
u^{(4)}=\lambda f(u) \quad \text { in }(0,1), \\
u(0)=a \geq 0, \quad u^{\prime}(0)=a^{\prime} \geq 0, \quad u(1)=b \geq 0, \quad u^{\prime}(1)=-b^{\prime} \leq 0 .
\end{array}\right.
$$
}

The methods employed are upper and lower solutions and degree theory arguments.

1. Introduction. In this paper we are concerned with the existence and multiplicities of positive solutions of the nonlinear fourth order problem

$$
\left\{\begin{array}{l}
u^{(4)}=\lambda f(u) \quad \text { in }(0,1), \\
u(0)=a, \quad u^{\prime}(0)=a^{\prime}, \quad u(1)=b, \quad u^{\prime}(1)=-b^{\prime} .
\end{array}\right.
$$

To emphasize the dependence on $\lambda$, we shall often denote the problem by $(1)_{\lambda}$, with $u_{\lambda}$ denoting a corresponding solution. Throughout this paper we assume, without further mention, that $\lambda$ is a positive parameter, $a, a^{\prime}, b, b^{\prime}$ are nonnegative constants, and $f$ is continuous on $[0, \infty)$ with $f:(0, \infty) \rightarrow$ $(0, \infty)$.

If we make the convention that $f(u)=f(0)$ when $u<0$, then by the maximum principle for fourth order inequalities given below, all nontrivial solutions $u \in C^{4}[0,1]$ of $(1)_{\lambda}$ are positive, i.e., $u(x)>0$ for $x \in(0,1)$.

Our main result is as follows:

THEOREM 1.1. Assume the following hypotheses:

$\left(\mathrm{H}_{1}\right) \quad f_{\infty} \equiv \lim _{u \rightarrow+\infty} \frac{f(u)}{u}=\infty$.

$\left(\mathrm{H}_{2}\right) \quad f_{0} \equiv \lim _{u \rightarrow 0} \frac{f(u)}{u}=\infty$.

2000 Mathematics Subject Classification: Primary 34B18.

Key words and phrases: nonlinear fourth order boundary value problems, multiplicity of positive solutions, upper and lower solutions, degree theory. 
$\left(\mathrm{H}_{3}\right) \quad f$ is nondecreasing.

Then there exists a positive number $\lambda^{*}$ such that $(1)_{\lambda}$ has at least two positive solutions for $0<\lambda<\lambda^{*}$, at least one positive solution for $\lambda=\lambda^{*}$ and no positive solutions for $\lambda>\lambda^{*}$.

In the case of a second order equation, analogous results have been proved under homogeneous boundary conditions with various hypotheses concerning the smoothness and growth properties of $f$. Among them, one may refer, with further references therein, to Dang, Schmitt and Shivaji [2], Ha and Lee [5] and Lin [7]. For systems of second order equations, see Dunninger and Wang [4].

The paper is organized as follows. In Section 2, some notation and preliminaries are introduced. Next, in Section 3, we use the method of upper and lower solutions to establish an existence result. In Section 4, the proof of the main theorem is given using degree theory arguments.

2. Preliminaries. Define

$$
T(\lambda, u)(x)=\varphi(x)+\lambda \int_{0}^{1} g(x, y) f(u(y)) d y
$$

where $\varphi$ satisfies

$$
\left\{\begin{array}{l}
\varphi^{(4)}=0 \quad \text { in }(0,1), \\
\varphi(0)=a, \quad \varphi^{\prime}(0)=a^{\prime}, \quad \varphi(1)=b, \quad \varphi^{\prime}(1)=-b^{\prime},
\end{array}\right.
$$

and $g(x, y)$ is Green's function given explicitly by

$$
g(x, y)= \begin{cases}x^{2}(1-y)^{2}(3 y-x-2 x y) / 6, & x \leq y \\ y^{2}(1-x)^{2}(3 x-y-2 x y) / 6, & x \geq y\end{cases}
$$

Then $(1)_{\lambda}$ is equivalent to the fixed point equation

$$
T(\lambda, u)=u
$$

in the usual Banach space $C[0,1]$ equipped with the sup norm. The operator $T:[0, \infty) \times C[0,1] \rightarrow C[0,1]$ is clearly completely continuous.

We shall need to make use of the following maximum principle [3]:

THEOREM 2.1. Let $u \in C^{4}[0,1]$ satisfy the differential inequality

$$
u^{(4)} \geq 0 \quad \text { in }(0,1) \text {. }
$$

(i) If $u(0) \geq 0, u^{\prime}(0) \geq 0, u(1) \geq 0, u^{\prime}(1) \leq 0$, then either $u>0$ or $u \equiv 0$ in $(0,1)$.

(ii) Let $u$ be nonconstant and satisfy $u^{\prime}(0)=u^{\prime}(1)=0$. If $u$ assumes its minimum value at $x=0$, then $u^{\prime \prime \prime}(0)<0$, whereas if $u$ assumes its minimum value at $x=1$, then $u^{\prime \prime \prime}(1)>0$. 
By reversing the above inequalities, we obtain analogous statements for the maximum value.

We shall need the following a priori estimate.

Lemma 2.2. Assume $\left(\mathrm{H}_{1}\right)$. For each $\mu>0$ there exists a positive constant $M$, which depends on $\mu$, such that $\|u\|_{3}<M$ for all solutions $u$ of $(1)_{\lambda}$ with $\lambda \geq \mu, \lambda$ in a compact interval. Here $\|u\|_{3}=\sum_{i=0}^{3} \max _{x \in[0,1]}\left|u^{i}(x)\right|$.

Proof. Denote by $\lambda_{1}$ the smallest positive eigenvalue of

$$
\left\{\begin{array}{l}
\phi^{(4)}=\lambda \phi \quad \text { in }(0,1) \\
\phi(0)=\phi^{\prime}(0)=\phi(1)=\phi^{\prime}(1)=0
\end{array}\right.
$$

with $\phi_{1}$ denoting the corresponding eigenfunction satisfying $\phi_{1}(x)>0$ for $x \in(0,1)$. In view of the above maximum principle, $\phi_{1}^{\prime \prime \prime}(0)<0$ and $\phi_{1}^{\prime \prime \prime}(1)$ $>0$, from which we can readily deduce that $\phi_{1}^{\prime \prime}(0)>0$ and $\phi_{1}^{\prime \prime}(1)>0$.

By $\left(\mathrm{H}_{1}\right)$, there is a positive constant $c_{2}$ such that $f(u) \geq c_{1} u-c_{2}$ for all $u \geq 0$, where $c_{1} \mu>\lambda_{1}$. Multiplying the equation in $(1)_{\lambda}$ by $\phi_{1}$ and integrating, we have

$$
\begin{aligned}
\lambda_{1} \int_{0}^{1} \phi_{1} u d x & =\int_{0}^{1} \phi_{1} u^{(4)} d x+\left[\phi_{1}^{\prime} u^{\prime \prime}-\phi_{1}^{\prime \prime} u^{\prime}+\phi_{1}^{\prime \prime \prime} u-\phi_{1} u^{\prime \prime \prime}\right]_{0}^{1} \\
& \geq \mu \int_{0}^{1} \phi_{1} f(u) d x+b^{\prime} \phi_{1}^{\prime \prime}(1)+a^{\prime} \phi_{1}^{\prime \prime}(0)+b \phi_{1}^{\prime \prime \prime}(1)-a \phi_{1}^{\prime \prime \prime}(0) \\
& \geq c_{1} \mu \int_{0}^{1} \phi_{1} u d x-c_{3}
\end{aligned}
$$

for some positive constant $c_{3}$. Consequently, it follows that

$$
\int_{0}^{1} \phi_{1} u d x \leq \frac{c_{3}}{c_{1} \mu-\lambda_{1}}, \quad \int_{0}^{1} \phi_{1} f(u) d x \leq c_{4}
$$

for some positive constant $c_{4}$.

Let $\varrho(y)=y^{2}(1-y)^{2}$. By a result obtained in [1], there is a positive constant $k$ such that

$$
0 \leq g(x, y) \leq k \varrho(y) \quad \text { for all } 0 \leq x, y \leq 1 .
$$

Furthermore, since $\phi_{1}^{\prime \prime}(0)>0, \phi_{1}^{\prime \prime}(1)>0, \varrho^{\prime \prime}(0)>0$ and $\varrho^{\prime \prime}(1)>0$, several applications of L'Hôpital's rule show that

$$
\lim _{x \rightarrow 0^{+}} \frac{\varrho(x)}{\phi_{1}(x)}>0, \quad \lim _{x \rightarrow 1^{-}} \frac{\varrho(x)}{\phi_{1}(x)}>0 .
$$

Hence, there are positive constants $k_{1}$ and $k_{2}$ such that

$$
k_{1} \varrho(x) \leq \phi_{1}(x) \leq k_{2} \varrho(x) \quad \text { for all } x \in[0,1] .
$$


Using (2), (3), (4) in the integral representation

$$
u(x)=\varphi(x)+\lambda \int_{0}^{1} g(x, y) f(u(y)) d y
$$

we deduce that the solutions $u_{\lambda}$ of $(1)_{\lambda}$ are a priori bounded in $C[0,1]$ for $\lambda \geq \mu, \lambda$ belonging to a compact interval. By using this a priori bound in (5), we see that the $u_{\lambda}$ are also a priori bounded in $C^{3}[0,1]$.

3. Existence. In order to prove the existence of positive solutions, we rely on the method of upper and lower solutions.

Consider the system

$$
\left\{\begin{array}{l}
u^{(4)}=F(u) \quad \text { in }(0,1), \\
u(0)=\gamma_{0}, \quad u^{\prime}(0)=\gamma_{0}^{\prime}, \quad u(1)=\gamma_{1}, \quad u^{\prime}(1)=\gamma_{1}^{\prime},
\end{array}\right.
$$

where $\gamma_{i}, \gamma_{i}^{\prime}(i=0,1)$ are given constants. A function $\bar{u} \in C^{4}[0,1]$ is called an upper solution of (6) if

$$
\left\{\begin{array}{l}
\bar{u}^{(4)} \geq F(\bar{u}) \quad \text { in }(0,1), \\
\bar{u}(0) \geq \gamma_{0}, \quad \bar{u}^{\prime}(0) \geq \gamma_{0}^{\prime}, \quad \bar{u}(1) \geq \gamma_{1}, \quad \bar{u}^{\prime}(1) \leq \gamma_{1}^{\prime} .
\end{array}\right.
$$

Similarly, a function $\underline{u} \in C^{4}[0,1]$ is called a lower solution of (6) if it satisfies the reverse of the above inequalities.

We define

$$
[\underline{u}, \bar{u}]=\left\{u \in C^{4}[0,1]: \underline{u}(x) \leq u(x) \leq \bar{u}(x), x \in[0,1]\right\} .
$$

THEOREM 3.1. Let $\bar{u}, \underline{u}$ be upper and lower solutions, respectively, of (6) such that $\underline{u}(x) \leq \bar{u}(x)$ for $x \in[0,1]$. Let $F \in C[\underline{u}, \bar{u}]$ be nondecreasing. Then (6) has a solution $u \in C^{4}[0,1]$ such that $\underline{u}(x) \leq u(x) \leq \bar{u}(x)$ for all $x \in[0,1]$.

Proof. For a given function $u \in C[0,1]$ define

$$
u^{*}(x)= \begin{cases}\bar{u}(x) & \text { if } u(x) \geq \bar{u}(x) \\ u(x) & \text { if } \underline{u}(x) \leq u(x) \leq \bar{u}(x) \\ \underline{u}(x) & \text { if } u(x) \leq \underline{u}(x)\end{cases}
$$

and set $F^{*}(x, u)=F\left(u^{*}(x)\right)$. Since the function $F^{*}$ is bounded and continuous on $(-\infty, \infty)$, an application of the Schauder fixed point theorem yields the existence of a solution $u \in C^{4}[0,1]$ of $u^{(4)}=F^{*}(x, u)$ satisfying the boundary conditions in (6). To complete the proof it suffices to show that $u \in[\underline{u}, \bar{u}]$, for then $u$ satisfies $u^{(4)}=F(u)$. To this end, we note that since $u^{*} \in[\underline{u}, \bar{u}]$ and $F$ is nondecreasing, $z=\bar{u}-u$ satisfies

$$
\left\{\begin{array}{l}
z^{(4)} \geq F(\bar{u})-F\left(u^{*}\right) \geq 0 \quad \text { in }(0,1) \\
z(0) \geq 0, \quad z^{\prime}(0) \geq 0, \quad z(1) \geq 0, \quad z^{\prime}(1) \leq 0 .
\end{array}\right.
$$


Hence by the maximum principle, $u(x) \leq \bar{u}(x)$ for $x \in[0,1]$. Similarly, we can show $\underline{u}(x) \leq u(x)$ for $x \in[0,1]$ and the proof is complete.

Let $\Gamma$ denote the set of $\lambda>0$ such that a positive solution of $(1)_{\lambda}$ exists, and let $\lambda^{*}=\sup \Gamma$. The main result of this section is as follows:

ThEOREM 3.2. Assume $\left(\mathrm{H}_{1}\right)-\left(\mathrm{H}_{3}\right)$. Then $(1)_{\lambda}$ has a positive solution $u_{\lambda}$ for all $0<\lambda \leq \lambda^{*}<\infty$. Moreover, for $\lambda<\lambda^{*}$, we have

$$
\left\{\begin{array}{l}
0<u_{\lambda}(x)<u_{\lambda^{*}}(x), \quad x \in(0,1) \\
u_{\lambda}^{\prime \prime \prime}(0)>u_{\lambda^{*}}^{\prime \prime \prime}(0), \quad u_{\lambda}^{\prime \prime \prime}(1)<u_{\lambda^{*}}^{\prime \prime \prime}(1),
\end{array}\right.
$$

where $u_{\lambda^{*}}$ is a solution of $(1)_{\lambda^{*}}$.

Proof. Let $e \in C^{4}[0,1]$ denote the unique positive solution of

$$
\left\{\begin{array}{l}
e^{(4)}=1 \quad \text { in }(0,1), \\
e(0)=a, \quad e^{\prime}(0)=a^{\prime}, \quad e(1)=b, \quad e^{\prime}(1)=-b^{\prime} .
\end{array}\right.
$$

Then $e^{(4)}=1 \geq \lambda f(e)$ if $\lambda$ is sufficiently small, and so $e$ is a positive upper solution of $(1)_{\lambda}$. In order to determine an appropriate lower solution we distinguish the following two cases:

(i) If 0 is not a solution of $(1)_{\lambda}$, then we can choose 0 as a lower solution of $(1)_{\lambda}$.

(ii) If 0 is a solution of $(1)_{\lambda}$ (in particular, this implies $a=a^{\prime}=b=b^{\prime}$ $=0)$, then we can choose $\varepsilon \phi_{1}$ as a positive lower solution of $(1)_{\lambda}$ for $\varepsilon>0$ sufficiently small. In fact, since $f_{0}=\infty$, we have

$$
\left(\varepsilon \phi_{1}\right)^{(4)}-\lambda f\left(\varepsilon \phi_{1}\right)=\varepsilon \phi_{1}\left(\lambda_{1}-\lambda \frac{f\left(\varepsilon \phi_{1}\right)}{\varepsilon \phi_{1}}\right) \leq 0
$$

for $\varepsilon>0$ sufficiently small. Moreover, since $e^{\prime \prime}(0)>0, e^{\prime \prime}(1)>0, \phi_{1}^{\prime \prime}(0)>0$ and $\phi_{1}^{\prime \prime}(1)>0$, several applications of L'Hôpital's rule show that

$$
\lim _{x \rightarrow 0^{+}} \frac{e(x)}{\phi_{1}(x)}>0, \quad \lim _{x \rightarrow 1^{-}} \frac{e(x)}{\phi_{1}(x)}>0 .
$$

Hence we can choose $\varepsilon$ even smaller, if necessary, so that $e(x) \geq \varepsilon \phi_{1}(x)$ for $x \in[0,1]$.

Thus in either case, Theorem 3.1 implies that $(1)_{\lambda}$ has a positive solution for $\lambda$ sufficiently small, i.e., $\lambda^{*}>0$.

Now let $u \in C^{4}[0,1]$ be a solution of $(1)_{\lambda}$. By $\left(\mathrm{H}_{1}\right)$ and $\left(\mathrm{H}_{2}\right)$, there is a constant $c>0$ such that $f(u) \geq c u$ for all $u \geq 0$. Thus by a computation, as in the proof of Lemma 2.2, we have

$$
\left(\lambda_{1}-c \lambda\right) \int_{0}^{1} \phi_{1} u d x \geq 0
$$


and by choosing $\lambda$ sufficiently large we see that a positive solution of $(1)_{\lambda}$ cannot exist. Hence $\lambda^{*}<\infty$.

To see that $\lambda^{*} \in \Gamma$, let $\lambda_{n} \rightarrow \lambda^{*}$ where $\lambda_{n} \in \Gamma$. By Lemma 2.2, the corresponding solutions $u_{n}$ of $(1)_{\lambda_{n}}$ are bounded in $C[0,1]$. Since the operator $T$ is compact, a standard limiting process shows that $\lambda^{*} \in \Gamma$.

Let $u_{\lambda^{*}}$ be a solution of $(1)_{\lambda^{*}}$ and let $\lambda<\lambda^{*}$. Then $u_{\lambda^{*}}$ is a positive upper solution of $(1)_{\lambda}$, and consequently, $(1)_{\lambda}$ has a positive solution $u_{\lambda} \leq u_{\lambda^{*}}$. Since $f$ is nondecreasing, it easily follows from the maximum principle that $0<u_{\lambda}(x)<u_{\lambda^{*}}(x)$ for $x \in(0,1), u_{\lambda}^{\prime \prime \prime}(0)>u_{\lambda^{*}}^{\prime \prime \prime}(0)$ and $u_{\lambda}^{\prime \prime \prime}(1)<u_{\lambda^{*}}^{\prime \prime \prime}(1)$.

\section{Main theorem. Define}

$$
\bar{f}(x, u)= \begin{cases}f\left(u_{\lambda^{*}}(x)\right) & \text { if } u \geq u_{\lambda^{*}}(x), \\ f(u) & \text { if } 0 \leq u \leq u_{\lambda^{*}}(x), \\ f(0) & \text { if } u \leq 0,\end{cases}
$$

and let $\bar{T}(\lambda, u)$ be the integral operator analogous to $T(\lambda, u)$ defined by $\bar{f}$. Since $\bar{f}$ is bounded, all solutions $u$ of $u=\bar{T}(\lambda, u)$ are a priori bounded in $C^{3}[0,1]$ for $\lambda$ belonging to a compact interval. Without loss of generality we can assume $\|u\|_{3}<M$, where $M>0$ is given by Lemma 2.2 .

Consider

$$
\begin{aligned}
\Omega=\left\{u \in C^{3}[0,1]:\|u\|_{3}<M, u<\right. & u_{\lambda^{*}} \text { in }(0,1), \\
& \left.u^{\prime \prime \prime}(0)>u_{\lambda^{*}}^{\prime \prime \prime}(0), u^{\prime \prime \prime}(1)<u_{\lambda^{*}}^{\prime \prime \prime}(1)\right\} .
\end{aligned}
$$

Then $\Omega$ is bounded and open in $C^{3}[0,1]$.

Lemma 4.1. Let $0<\lambda<\lambda^{*}$. If $u$ is a solution of $u=\bar{T}(\lambda, u)$, then $u \in \Omega$.

Proof. First note that

$$
\left(u-u_{\lambda^{*}}\right)^{(4)} \leq \lambda\left(\bar{f}(x, u)-f\left(u_{\lambda^{*}}\right)\right) \quad \text { in }(0,1) .
$$

At all points $x \in(0,1)$ where $u(x) \geq u_{\lambda^{*}}(x)$,

$$
\bar{f}(x, u)-f\left(u_{\lambda^{*}}\right)=f\left(u_{\lambda^{*}}\right)-f\left(u_{\lambda^{*}}\right)=0 .
$$

On the other hand, at all points $x \in(0,1)$ where $u(x) \leq u_{\lambda^{*}}(x)$, the monotonicity of $f$ implies

$$
\bar{f}(x, u)-f\left(u_{\lambda^{*}}\right)=f(u)-f\left(u_{\lambda^{*}}\right) \leq 0 .
$$

Hence we have

$$
\left\{\begin{array}{l}
\left(u-u_{\lambda^{*}}\right)^{(4)} \leq 0 \quad \text { in }(0,1) \\
\left(u-u_{\lambda^{*}}\right)(0)=\left(u-u_{\lambda^{*}}\right)^{\prime}(0)=\left(u-u_{\lambda^{*}}\right)(1)=\left(u-u_{\lambda^{*}}\right)^{\prime}(1)=0
\end{array}\right.
$$

which, by the maximum principle, yields $u \in \Omega$.

Proof of Theorem 1.1. Let $0<\lambda<\lambda^{*}$. By Theorem 3.2, there exists a solution $u_{\lambda}$ of $(1)_{\lambda}$, and moreover, $u_{\lambda} \in \Omega$. Since $\bar{T}$ is bounded for $\lambda$ in 
compact intervals,

$$
d\left(I-\bar{T}(\lambda, \cdot), B\left(u_{\lambda}, R\right), 0\right)=1
$$

if $R$ is sufficiently large, where $B\left(u_{\lambda}, R\right)$ is the ball centered at $u_{\lambda}$ with radius $R$ in $C^{3}[0,1]$. By Lemma $4.1, d(I-\bar{T}(\lambda, \cdot), \Omega, 0)$ is defined. Moreover, since $\bar{T}(\lambda, \cdot)$ has no fixed point in $B\left(u_{\lambda}, R\right) \backslash \Omega$, by the excision property of degree we have

$$
d(I-T(\lambda, \cdot), \Omega, 0)=d(I-\bar{T}(\lambda, \cdot), \Omega, 0)=1 .
$$

On the other hand, it follows from Lemma 2.2 that for $\mu>0$ there exists an $M>0$ such that for $\lambda \geq \mu, \lambda$ in compact intervals,

$$
d(I-T(\lambda, \cdot), B(0, M), 0)=\text { constant }
$$

where $B(0, M)$ is the ball centered at 0 with radius $M$ in $C^{3}[0,1]$. The latter degree must equal 0 since $(1)_{\lambda}$ has no solutions for $\lambda>\lambda^{*}$. Hence by the excision property of degree,

$$
d(I-T(\lambda, \cdot), B(0, M) \backslash \bar{\Omega}, 0)=-1
$$

and the existence of a second positive solution to $(1)_{\lambda}$ follows.

REMARK. The above analysis can be readily extended to problems of the form

$$
\left\{\begin{array}{l}
u^{(4)}=\lambda f(u) \quad \text { in }(0,1), \\
u(0)=a \geq 0, \quad u^{\prime \prime}(0)=a^{\prime} \leq 0, \quad u(1)=b \geq 0, \quad u^{\prime \prime}(1)=-b^{\prime} \leq 0,
\end{array}\right.
$$

and thus provides an improvement and generalization of a related result in $[6]$.

I would like to thank the referee for several helpful suggestions which improved the presentation of this paper.

\section{References}

[1] R. Dalmasso, Elliptic equations of order $2 m$ in annular domains, Trans. Amer. Math. Soc. 347 (1995), 3575-3585.

[2] H. Dang, K. Schmitt and R. Shivaji, On the number of solutions of boundary value problems involving the p-Laplacian, Electron. J. Differential Equations 1 (1996), 1-9.

[3] D. R. Dunninger, Maximum principles for fourth order ordinary differential inequalities, J. Math. Anal. Appl. 82 (1981), 399-405.

[4] D. R. Dunninger and H. Wang, Multiplicity of positive radial solutions for an elliptic system on an annulus, Nonlinear Anal. 42 (2000), 803-811.

[5] K. S. Ha and Y. Lee, Existence of multiple positive solutions of singular boundary value problems, ibid. 28 (1997), 1429-1438.

[6] G. Hernandez and R. Manasevich, Existence and multiplicity of solutions of a fourth order equation, Appl. Anal. 54 (1994), 237-250. 
[7] S. S. Lin, Positive radial solutions and nonradial bifurcation for semilinear elliptic equations in annular domains, J. Differential Equations 86 (1990), 367-391.

Department of Mathematics

Michigan State University

East Lansing, MI 48824, U.S.A.

E-mail: dun@math.msu.edu

Reçu par la Rédaction le 28.6.2000

Révisé le 10.1.2001 et 19.4.2001 